\title{
The Effects of Serine Palmitoyltransferase Inhibitor, ISP-I, on UV-Induced Barrier Disruption in the Stratum Corneum
}

\author{
Koji Mizukoshi, ${ }^{*}, a$ Hiroshi Oshima, ${ }^{a}$ Katsuo Matsumoto, ${ }^{a}$ Ryouji Hirose, ${ }^{b}$ and Tetsuro Fustta ${ }^{c}$

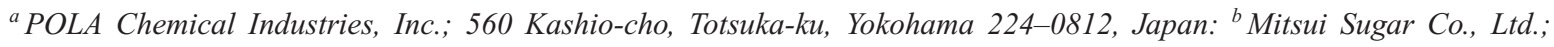 \\ 2-8-2 Nihonbashi-Honcho, Chuo-ku, Tokyo 103-8423, Japan: and ${ }^{c}$ Research Institute for Production Development; 15 \\ Morimoto-cho, Shimogamo, Sakyo-ku, Kyoto 606-0805, Japan. \\ Received May 23, 2010; accepted September 4, 2010; published online September 8, 2010
}

We examined the effects of ISP-I (myriocin, thermozymocidin) - a potent inhibitor of serine palmitoyltransferase (SPT) which is involved in the ceramide synthetic pathway-on skin barrier function in post-UVB-irradiated hairless mouse skin. Disruption of the skin barrier function after UVB irradiation as represented by the increase in transepidermal water loss (TEWL) was significantly suppressed with ISP-I treatment. In the ISP-Itreated skin, the peak of cell proliferation was observed $24 \mathrm{~h}$ earlier than in vehicle-treated skin. In addition, the number of apoptotic cells in ISP-I-treated skin showed a sharp decrease at 48 and $72 \mathrm{~h}$ post-irradiation. The number of stratum corneum cell layers was increased in ISP-I-treated skin at $\mathbf{7 2} \mathrm{h}$ after UVB irradiation; at this time, TEWL in ISP-I-treated skin was lower than that in the vehicle-treated skin. We suggest ISP-I treatment altered cell proliferation and apoptosis after UVB exposure by modulating ceramide synthesis in epidermal cells, resulting in an increase of stratum corneum layers which lessened the effects of irradiation-induced barrier disruption.

Key words ISP-I (myriocin); serine palmitoyltransferase; UVB; ceramide; skin barrier

The intercellular lipids of the stratum corneum, referred to as the "mortar" in the brick-and-mortar model, contain ceramides which play an essential role in the barrier function of the stratum corneum. ${ }^{1)}$ Ceramides are also involved in signal transduction pathways that are linked to cell proliferation ${ }^{2,3)}$ and apoptosis in various cell types. ${ }^{4,5)}$ Thus, ceramides not only function as an intercellular component of the skin barrier but also affect cell activity.

The first step of de novo biosynthesis of ceramides is catalyzed by serine palmitoyltransferase (SPT), which is a condensation reaction between L-serine and palmitoyl-CoA. It has been suggested that SPT is a key enzyme in regulating sphingolipid levels involved in ceramide production. ${ }^{\text {) }}$

The immunosuppressant, ISP-I, was isolated by Fujita et $a l^{7)}$ from the culture medium of Isaria sinclairii, a fungus found on cicada larva. The structure is identical to the antifungal agents myriocin ${ }^{8,9)}$ and thermozymocidin, ${ }^{10,11)}$ which were isolated from Myriococcum albomyces and Mycelia sterilia, respectively. Miyake et al. elucidated that ISP-I inhibits SPT. ${ }^{12)}$

The effects of SPT inhibitors on the barrier function of the stratum corneum have been examined previously, and involved tape-stripping to physically remove the stratum corneum, or an organic solvent to extract intercellular lipids. ${ }^{13,14)}$ These methods are suitable in assessing the effects of SPT inhibition on the de novo synthesis of ceramides that are released into intercellular spaces after tape stripping or organic solvent treatment. However, these methods are not suitable for assessing the effect of ceramides on cellular proliferation or apoptosis.

In the present study, we employed UVB irradiation which alters cellular proliferation and apoptosis, and leads to barrier disruption. ${ }^{15,16)}$ We investigated the effects of ISP-I treatment on the barrier function of skin by inhibiting ceramide synthesis.

\section{MATERIALS AND METHODS}

Materials ISP-I ${ }^{17)}$ (Fig. 1) was isolated from the culture broth of M. sterilia. Dispase was purchased from Godo Shusei Co., Ltd. (Tokyo, Japan) and trypsin was purchased from Wako Pure Chemical Industries (Osaka, Japan). Anti-5bromo-2'-deoxyuridine (BrdU) monoclonal antibody and BrdU were included in the BrdU assay kit from Cosmo Bio Co., Ltd. (Tokyo, Japan). Apoptotic cells were stained and detected by the TdT-mediated deoxyuridine triphosphate (dUTP)-biotin nick end-labeling method (TUNEL) using a TACS $^{\mathrm{TM}}$ 2TdT/Blue Label kit (Cosmo Bio Co., Ltd., Tokyo, Japan). The ceramide standards for the detection of ceramide-1 using thin-layer chromatography were purchased from Merck (Whitehouse Station, NJ, U.S.A.). Cellophane tape used for tape stripping of the stratum corneum was purchased from Sekisui Chemical Co., Ltd. (Osaka, Japan).

Animals Female hairless mice (Hr-Kud, 8 weeks old) were purchased from Kyudo Co., Ltd. (Kumamoto, Japan). They were kept in an environment with a constant temperature and humidity with a 12-h light/dark cycle, and were fed a standard diet and given water ad libitum. All experiments were approved by an in-house animal ethics committee.

Measurement of Transepidermal Water Loss Transepidermal water loss (TEWL) was measured at various time points with a TEWA meter (TM 120, Courage and Khazaka, Köln, Germany).

The Effect of ISP-I on Barrier Recovery after TapeStripping of the Stratum Corneum A barrier disruption model was established by conducting five to seven rounds of tape stripping on the dorsal skin until the TEWL value was

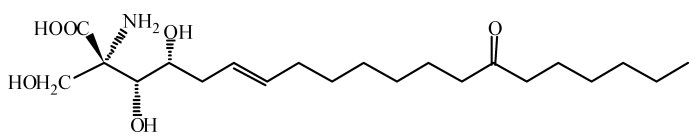

Fig. 1. Chemical Structure of ISP-I 
approximately $60 \mathrm{~g} / \mathrm{m}^{2} / \mathrm{h}$. ${ }^{13)}$ ISP-I solution $(100 \mu \mathrm{l}$ of $0.02 \%$ ISP-I in $80 \%$ ethanol) was applied to the $4-\mathrm{cm}^{2}$ tape-stripped site at $0,1,3,6,12,24,48,72$ and $96 \mathrm{~h}$ after tape stripping. The vehicle solution $(80 \%$ ethanol, v/v) was applied to the control group. TEWL was measured before and at $0,3,6,12$, $24,48,72,96$ and $120 \mathrm{~h}$ after tape stripping to assess epidermal barrier function.

The Effect of ISP-I Treatment on Barrier Recovery in Acetone-Treated Skin A separate barrier disruption model was established by treating hairless mouse dorsal skin with cotton soaked in acetone until the TEWL value was approximately $60 \mathrm{~g} / \mathrm{m}^{2} / \mathrm{h}^{14)}$ A solution of ISP-I (100 $\left.\mu \mathrm{l}\right)$ was applied to the $4-\mathrm{cm}^{2}$ acetone-treated site at $0,1,3,6,12,24,48,72$ and $96 \mathrm{~h}$ after acetone treatment. The vehicle solution $(80 \%$ ethanol, v/v) was applied to the control group. TEWL was measured before and at $0,3,6,12,24,48,72,96$ and $120 \mathrm{~h}$ after acetone treatment to assess epidermal barrier function.

The Effect of ISP-I Treatment on Barrier Function in UVB-Irradiated Hairless Mouse Skin Dorsal skin of hairless mouse was irradiated with a single UVB dose of $100 \mathrm{~mJ} / \mathrm{cm}^{2}$ (2 minimum erythema dose (MED)) using UV lamps (FL20S.E-30/DMR, Toshiba, Tokyo, Japan). ISP-I solution ( $100 \mu \mathrm{l}$ of $0.02 \%$ ISP-I in $80 \%$ ethanol) was applied to the 4-cm $\mathrm{cm}^{2}$ UVB-treated area at $0,2,4,6,12,24,48$ and $72 \mathrm{~h}$ after UVB irradiation. The vehicle solution $(80 \%$ ethanol, $\mathrm{v} / \mathrm{v})$ was applied to the control group. TEWL was measured before and at $0,24,48,72,96,120$ and $168 \mathrm{~h}$ after UVB irradiation to assess epidermal barrier function.

Proliferation Assay of Epidermal Basal Cells The UVB-irradiated epidermis was subjected to the 5-bromo- $2^{\prime}$ deoxyuridine (BrdU) assay. Briefly, BrdU solution (1.5 mg in phosphate buffered saline (PBS)) was injected into the peritoneal cavity of mice before and 24,48 and $72 \mathrm{~h}$ after UVB irradiation. The mice were sacrificed and skin samples were obtained $20 \mathrm{~min}$ after each injection. ${ }^{18)}$ Skins were snap frozen and embedded in OCT compound (Tissue-Tek O.C.T. Compound, Sakura Finetek, Tokyo, Japan). Cryosections were prepared at $6 \mu \mathrm{m}$, then incubated with an anti-BrdU monoclonal antibody followed by diaminobenzidine staining. The stained sections were observed through a microscope at 200 magnification with micrometer, and BrdU-positive basal cells were counted in five random fields per specimen. The number of BrdU positive cells per 1-mm length of the basal cell layer was determined.

Measurement of Apoptotic Cells in the Epidermis Frozen skin sections were prepared from the skin before and 24, 48 and $72 \mathrm{~h}$ after UVB irradiation, and subjected to TUNEL staining. Stained sections were observed through a microscope at 200 magnification with micrometer and TUNEL-positive cells (i.e., apoptotic cells) in the epidermis were counted in five random fields per specimen. The number of TUNEL positive cells per 1-mm length of the basal cell layer was determined.

Ceramide Content in the Stratum Corneum Skin samples were successively treated with dispase and trypsin to remove the dermis as well as living epidermal cells from the stratum corneum sheets, which were then lyophilized and their dry weights measured. ${ }^{19)}$ The method of lipid extraction from the stratum corneum and quantification of ceramide-1 were based on a previous report. ${ }^{20)}$ Briefly, intercellular lipids were extracted from the dried stratum corneum by successive treatments with chloroform-ethanol in the ratios of $2: 1,1: 1$ and $1: 2$. The collected solvents were evaporated and the lipid residues were kept under nitrogen. Thin layer chromatograms were developed with a mixture of chloroform, methanol and acetic acid (190:9:1) to separate ceramides from other lipids. After solvent development, the chromatograms were air-dried, sprayed with $50 \% \mathrm{H}_{2} \mathrm{SO}_{4}$ aqueous solution and colored on a hot plate. The colored lipids were quantified by photo-densitometry and the data were subjected to 2-dimensional image analysis. The amount of ceramides was quantified against ceramide standards.

Measurement of the Number of Stratum Corneum Layers The number of stratum corneum layers was measured based on a previous report. ${ }^{21)}$ Briefly, $1 \mathrm{~N} \mathrm{NaOH}$ solution was dripped onto $5-\mu \mathrm{m}$-thick frozen skin sections after air drying, and left for a few minutes. The number of stratum corneum layers was assessed by counting six random fields per specimen with a phase contrast light microscope at 200 magnification.

Statistical Analysis Statistical differences among different groups were evaluated using JMP software (Version 6, SAS Institute, U.S.A.). A $p$-value $<0.05$ was considered to be significant.

\section{RESULTS}

The Effect of ISP-I on Barrier Recovery in the TapeStripped and Acetone-Treated Skin We first examined how ISP-I treatment affects barrier recovery after tape stripping or acetone treatment in hairless mouse skin. After tape stripping or acetone treatment, recovery of barrier function was demonstrated by a decrease in TEWL value. ISP-I treatment resulted in a tendency towards a delay in barrier recovery; this was evident from $48 \mathrm{~h}$ post-treatment as demonstrated by the slower decline in TEWL value compared to control (Figs. 2, 3).

The Effect of ISP-I on Barrier Recovery after UVB Irradiation The TEWL peaked at $72 \mathrm{~h}$ after UVB irradiation of hairless mouse skin. However, when ISP-I was applied onto the skin immediately after irradiation, the increase in TEWL at $72 \mathrm{~h}$ was significantly suppressed compared to control (Fig. 4).

Cell proliferation was indicated by BrdU-positive cells. In vehicle-treated skin, it increased with time, and was accompanied by an increase in TEWL value after UVB irradiation. In ISP-I-treated skin, the number of BrdU-positive cells was

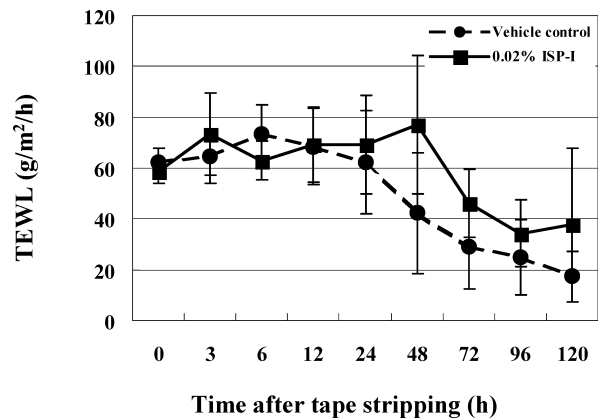

Fig. 2. Time Course of TEWL in ISP-I-Treated Hairless Mouse Skin after Tape Stripping

Values represent the mean of five animals ( \pm S.D.). 


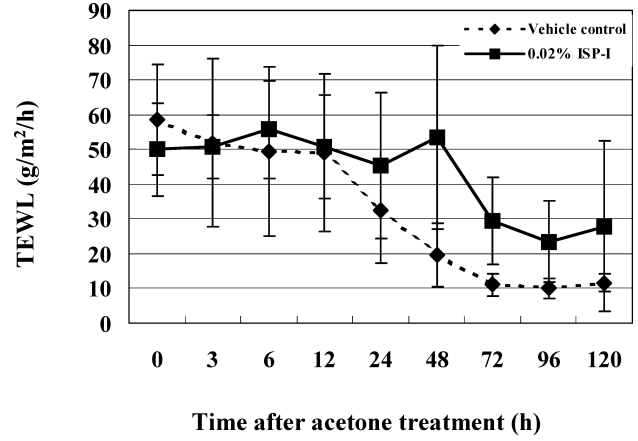

Fig. 3. Time Course of TEWL in ISP-I-Treated Hairless Mouse Skin after Acetone Treatment

Values represent the mean of five animals ( \pm S.D.).

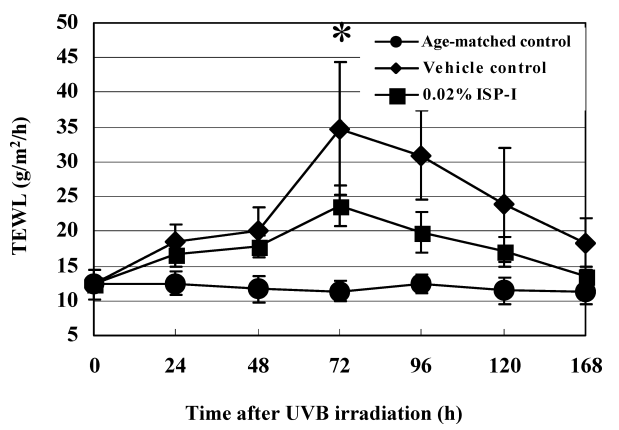

Fig. 4. Time Course of TEWL in ISP-I-Treated Hairless Mouse Skin after UVB Irradiation

TEWL was significantly suppressed in ISP-I-treated skin compared to vehicle control at $72 \mathrm{~h}$ post-irradiation $(* p<0.05, t$-test). Values represent the mean of five animals ( \pm S.D.).

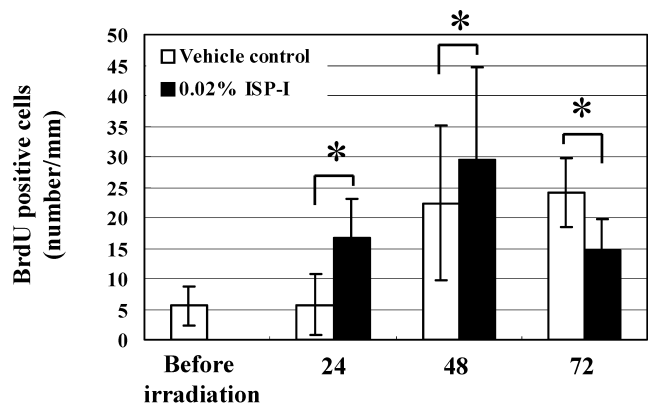

Time after UVB irradiation (h)

Fig. 5. Quantification of BrdU-Positive Epidermal Basal Cells in UVB-Irradiated Hairless Mouse Skin with or without ISP-I Treatment $(* p<0.05, t$ Test)

Values represent the mean of five animals ( \pm S.D. $)$.

significantly higher than that in vehicle-treated skin at 24 and $48 \mathrm{~h}$, but was significantly lower at $72 \mathrm{~h}$ (Fig. 5). The number of TUNEL-positive cells, which indicates apoptotic cells, markedly increased $24 \mathrm{~h}$ after irradiation in the control group, and then decreased over time (48, $72 \mathrm{~h}$ ). ISP-I-treated skin showed a similar tendency, but the number of TUNELpositive cells was significantly lower at 48 and $72 \mathrm{~h} \mathrm{com-}$ pared to control (Fig. 6).

The amount of ceramide-1 decreased in both ISP-I and control groups, and there was no significant difference between the two groups at any time (Fig. 7). The number of stratum corneum layers was counted using the alkali expan-

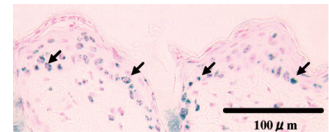

Vehicle-treated skin

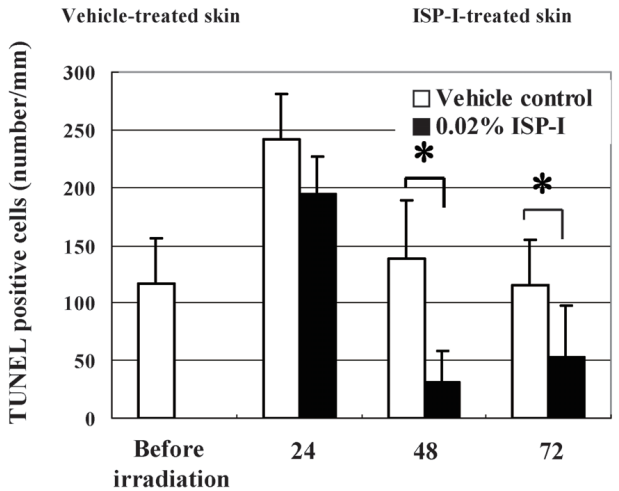

Time after UVB irradiation (h)

Fig. 6. Quantification of TUNEL-Positive Cells in the UVB-Irradiated Hairless Mouse Epidermis with or without ISP-I Treatment

Photos show TUNEL-positive cells in the vehicle-treated skin (left) and in the ISP-Itreated skin (right) at $48 \mathrm{~h}$ post-irradiation. Note that the number of TUNEL-positive cells were markedly decreased in the ISP-I-treated skin epidermis relative to control $(* p<0.05, t$-test). Values represent the mean of five animals ( \pm S.D.)

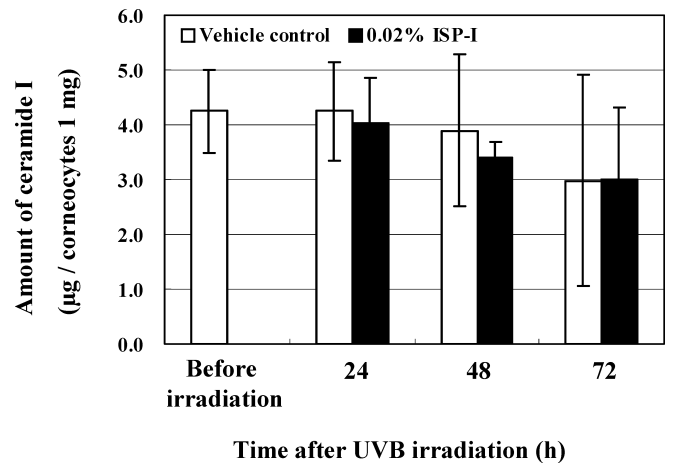

Fig. 7. Ceramide Amount in the Hairless Mouse Stratum Corneum with or without ISP-I Treatment after UVB Irradiation

Values represent the mean of five animals ( \pm S.D.).
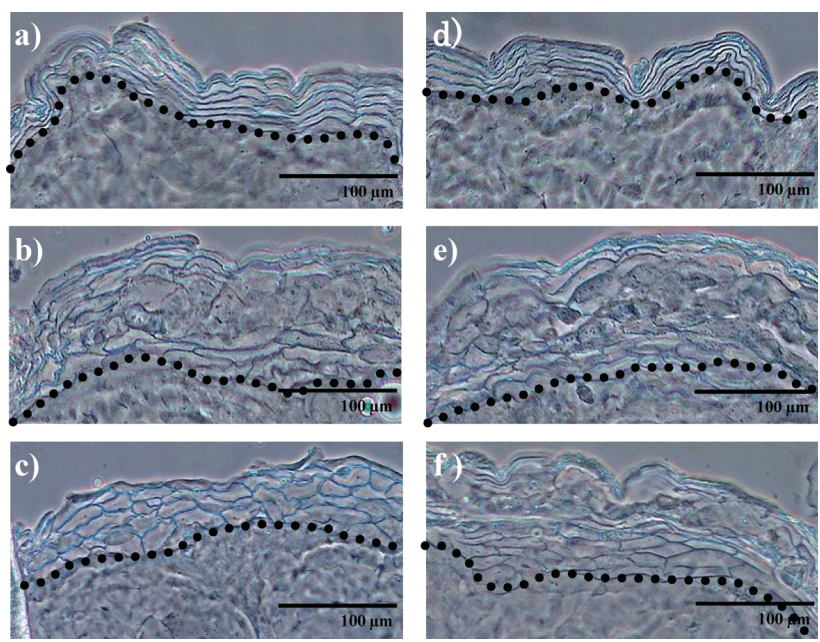

Fig. 8. The Effects of ISP-I Treatment on Stratification of Stratum Corneum

Vehicle-treated skin at a) $24 \mathrm{~h}$, b) $48 \mathrm{~h}$ and c) $72 \mathrm{~h}$ after UVB irradiation. ISP-Itreated skin at d) $24 \mathrm{~h}$, e) $48 \mathrm{~h}$ and f) $72 \mathrm{~h}$ after UVB irradiation. Dotted lines indicate the interface between stratum corneum and stratum granulosum. 


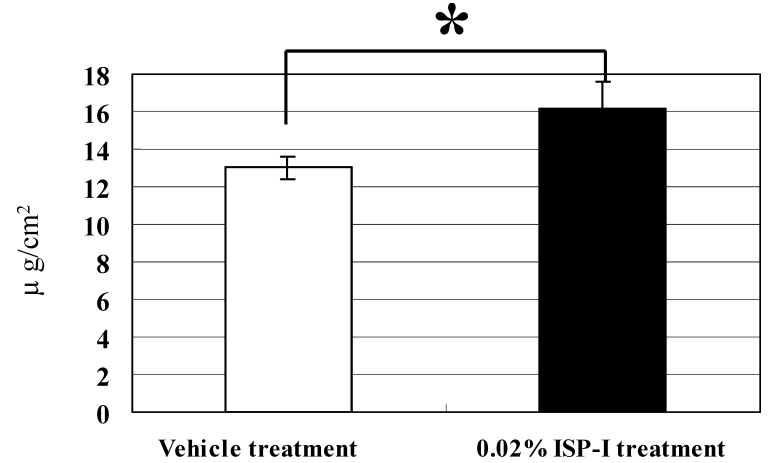

Fig. 9. Dry Weight of the Stratum Corneum in Skin Treated with or without ISP-I at $72 \mathrm{~h}$ after UVB Irradiation $(* p<0.05, t$-Test)

Values represent the mean of five animals ( \pm S.D. $)$.

sion method. There was no difference between the vehicle and ISP-I-treated groups at $24 \mathrm{~h}$, but it became higher in the ISP-I-treated skin at $72 \mathrm{~h}$ after UVB irradiation (Fig. 8). The dry weight of the stratum corneum in ISP-I-treated skin was significantly higher than that of vehicle-treated skin at $72 \mathrm{~h}$ after UVB irradiation (Fig. 9).

\section{DISCUSSION}

Intercellular lipids are composed of ceramides, cholesterol and fatty acids, and they form lamellar structures in the stratum corneum. It is known that changes in lipid composition lead to barrier impairment. ${ }^{22}$ ) Many studies have focused on ceramides as one of the intercellular lipid components that contribute to the barrier function of the skin. Holleran et $a l .{ }^{14)}$ reported that topical application of an inhibitor of ceramide synthesis caused a delay in barrier recovery after acetone treatment. In our study, we also confirmed that the application of ISP-I, an inhibitor of SPT, resulted in a tendency towards a delay in barrier recovery after acetone or tape stripping treatment. From this, we speculate that ISP-I influenced barrier recovery by inhibiting the de novo synthesis of ceramides.

Apart from the contribution of ceramides to barrier function, there have been studies which focused on the role of ceramides as intracellular messengers in signal transduction which affect cell proliferation and apoptosis. ${ }^{2-5)}$ Previous barrier disruption methods, namely tape stripping and acetone extraction treatment which removed the stratum corneum and/or the intercellular lipids, were used in studies on barrier function. While these methods are suitable for assessing the effects of SPT inhibition on the de novo synthesis of ceramides - which are released into the intercellular space after tape stripping or acetone treatment - they are not appropriate for investigating the role of ceramides in cell proliferation or apoptosis. For this purpose, we chose UVB irradiation as a method of barrier disruption that has been shown to influence epidermal barrier function, proliferation ${ }^{23)}$ and apoptosis ${ }^{24)}$ to study the effects of ISP-I.

As indicated by the TEWL increase, 2 MED of UVB caused barrier impairment in the vehicle and ISP-I-treated groups $72 \mathrm{~h}$ after irradiation (Fig. 4). We expected that ISP-I would delay barrier recovery and increase TEWL in a similar fashion to that observed in the tape stripping or acetone treatment experiments (Figs. 2, 3) at $72 \mathrm{~h}$ after irradiation as ISP-I is known to inhibit the action of SPT. However, ISP-I application significantly suppressed the increase in TEWL value compared to the TEWL value in the vehicle-treated skin after UVB irradiation (Fig. 4). There was no sign of skin roughness such as scaling in the treated hairless mouse (data not shown). These results appear to contradict the fact that ISP-I inhibits synthesis of ceramides, which play an essential role in the barrier function of the stratum corneum. ${ }^{1)}$

On the other hand, the number of stratum corneum cell layers increased in ISP-I-treated skin compared to vehicletreated skin, and the dry weight of the stratum corneum increased in ISP-I-treated skin as well at $72 \mathrm{~h}$ after UVB irradiation, when the barrier impairment was at its peak (Fig. 9). These findings suggest that the suppressive effects of ISP-I on the increase of TEWL at $72 \mathrm{~h}$ after UVB irradiation are due to the increase in the number of stratum corneum cell layers.

As the formation and desquamation of corneocyte is based on the balance between proliferation and differentiation of keratinocyte, ${ }^{25)}$ we currently suppose there would be some alterations in the corneocytes formed through the terminal differentiation process eventually affected by the shift of the peak in the cell proliferation (Fig. 5) and marked decrease of the number of apoptotic cells to the much lower level of control skin (Fig. 6) by the ISP-I treatment. Uchida et al. reported $^{26)}$ that UVB irradiation-induced apoptosis in cultured keratinocytes was significantly inhibited when the ceramidedependent pathway was inhibited by ISP-I treatment. Therefore, we tentatively suppose there would be some effects of ISP-I on the ceramide synthesis and signal transduction at the basal cell level even in the UVB irradiated skin although there was not marked difference in the amount of ceramides in the vehicle-treated and the ISP-I-treated, UVB irradiated skins at the stratum corneum level (Fig. 7). Identifying the alterations will contribute to the explanation of the increase in the number of stratum corneum and the decrease on TEWL.

Acknowledgements The authors thank Dr. Iizuka and Dr. Ishida-Yamamoto (Department of Dermatology, Asahikawa Medical College) for insightful comments and suggestions.

\section{REFERENCES}

1) Elias P. M., Feingold K. R., Semin. Dermatol., 11, 176-182 (1992).

2) Geilen C. C., Barz S., Bektas M., Skin Pharmacol. Appl. Skin Physiol., 14, 261-271 (2001).

3) Wakita H., Tokura Y., Yagi H., Nishimura K., Furukawa F., Takigawa M., Arch. Dermatol. Res., 286, 350-354 (1994).

4) Takeda S., Mitsutake S., Tsuji K., Igarashi Y., J. Biochem., 139, 255 262 (2006).

5) Ruvolo P. P., Leukemia, 15, 1153-1160 (2001).

6) Hanada K., Biochim. Biophys. Acta, 1632, 16-30 (2003).

7) Fujita T., Inoue K., Yamamoto S., Ikumoto T., Sasaki S., Toyama R., Chiba K., Hoshino Y., Okumoto T., J. Antibiot. (Tokyo), 47, 208-215 (1994).

8) Kluepfel D., Bagli J., Baker H., Charest M. P., Kudelski A., J. Antibiot. (Tokyo), 25, 109-115 (1972).

9) St-Jacques M., J. Org. Chem., 38, 1253-1260 (1973).

10) Craveri R., Manachini P. L., Aragozzini F., Experientia, 28, 867-868 (1972).

11) Aragozzini F, Manachini P. L., Craveri R., Rindone B., Scolastico C., Tetrahedron, 28, 5493-5498 (1972).

12) Miyake Y., Kozutsumi Y., Nakamura S., Fujita T., Kawasaki T., Biochem. Biophys. Res. Commun., 211, 396-403 (1995). 
13) Yang L., Mao-Qiang M., Taljebini M., Elias P. M., Feingold K. R., Br. J. Dermatol., 133, 679-685 (1995).

14) Holleran W. M., Man M. Q., Gao W. N., Menon G. K., Elias P. M., Feingold K. R., J. Clin. Invest., 88, 1338-1345 (1991).

15) Haratake A., Uchida Y., Schmuth M., Tanno O., Yasuda R., Epstein J. H., Elias P. M., Holleran W. M., J. Invest. Dermatol., 108, 769-775 (1997).

16) Kane K. S., Maytin E. V., J. Invest. Dermatol., 104, 62 -67 (1995).

17) Hanada K., Nishijima M., Fujita T., Kobayashi S., Biochem. Pharmacol., 59, 1211-1216 (2000).

18) Nakano R., Tsuyama S., Murata F., J. Dermatol. Sci., 14, 54-62 (1997).

19) Kitano Y., Okada N., Br. J. Dermatol., 108, 555-560 (1983).

20) Behne M., Uchida Y., Seki T., de Montellano P. O., Elias P. M.,
Holleran W. M., J. Invest. Dermatol., 114, 185-192 (2000).

21) Ya-Xian Z., Suetake T., Tagami H., Arch. Dermatol. Res., 291, 555559 (1999).

22) Lavrijsen A. P., Bouwstra J. A., Gooris G. S., Weerheim A., Boddé H. E., Ponec M., J. Invest. Dermatol., 105, 619-624 (1995).

23) Haratake A., Uchida Y., Mimura K., Elias P. M., Holleran W. M., J. Invest. Dermatol., 108, 319-323 (1997).

24) Baba T., Hanada K,, Hashimoto I., J. Dermatol. Sci., 12, 18-23 (1996).

25) Houben E., Paepe K. De., Rogiers V., Skin Pharmacol. Physiol., 20, $122-132$ (2007).

26) Uchida Y., Nardo A. D., Collins V., Elias P. M., Holleran W. M., J. Invest. Dermatol., 120, 662-669 (2003). 